\title{
CONTINUUM ELECTROSTATICS INVESTIGATION OF IONIC MICELLES USING ATOMISTIC MODELS
}

\section{S. Farafonov, ${ }^{\star}$ A. V. Lebed, N. O. Mchedlov-Petrossyan}

V. N. Karazin Kharkiv National University, Svobody sq., 4, Kharkiv, 61022, Ukraine *e-mail:farafonov@karazin.ua

The key parameter related to the structure of the electric double layer of ionic surfactant micelles - electrostatic potential - is considered. A brief overview of experimental methods and theoretical models for estimating electrostatic potential is given. The calculating method for the electrostatic potential based on a numerical solution of the Poisson-Boltzmann equation using an atomistic model of anionic surfactant micelle is proposed. The parameters necessary for the construction of atomistic models are obtained from molecular dynamic modeling. The electrostatic potentials for the micelles of sodium dodecyl sulfate and cetyltrimethylammonium bromide at different ionic strengths were calculated by this method. The results are discussed in comparison with the values calculated in the simplified model, the Ohshima - Healy - White equation.

Keywords: surfactant micelle, Stern layer, electrostatic potential, Poisson - Boltzmann equation, solvent-accessible surface.

INTRODUCTION. Micelles of colloidal surfactants are widely used in different fields of chemistry and related sciences and technologies [1-3]. Aqueous micellar solutions are almost transparent and thermodynamically stable (i.e., reversible) liquid systems [3-5]. They may be considered as the most simple and at the same time most important and unique representatives of the so-called organized solutions $[6,7]$. Hereafter, we shall consider ionic surfactants, which are diphilic molecules consisting of a hydrophobic portion and ionic group. In fact, they are colloidal electrolytes with a hydrocarbon chain containing from 8 to 18 carbon atoms.
Micelles of a given colloidal surfactant are formed in aqueous solutions upon reaching a certain concentration, the so-called critical micelle concentration (CMC) and at a temperature equal or above the so-called Krafft point. The driving force of micellization in water is the hydrophobic interaction [3-5]. The hydrophilic parts of the surfactants are directed towards the bulk (aqueous) phase, while the hydrophobic tails form the internal hydrocarbon core. According to the generally accepted model, these surfactant aggregates are highly porous, highly hydrated, disordered clusters in a state of dynamic equilibrium with monomers in the bulk phase. In the concentration range 
about the $\mathrm{CMC}$, the micelles are several nmsized and practically sphere-shaped.

Although many models of the structure of micelles of ionic surfactants can be found in the literature, they all agree on the main point. Namely, an electric double layer (EDL) appears at the micelle-water interface. The ionic head groups form the inner layer characterized by surface electrostatic potential $\Psi_{0}$. It attracts the counterions from solution, which form the outer layer. Different approaches were proposed for its description, starting from the Goüy Chapman model, where the counterions are considered size-less and adopting Boltzmann distribution of concentration immediately after the inner layer, and then the Stern model, where some portion of counterions is assumed to adsorb strongly and form a thin dense layer (subsequently called Stern layer), while Boltzmann distribution starts from its outer boundary. The thickness of the Stern layer is usually denoted as $\delta$, and the electrostatic potential at its boundary is hence denoted as $\Psi_{\delta}$. Various departures from this model were developed beginning with the classical papers by Stigter $[8,9]$. For the counterions in the Stern layer, mobile and localized adsorption models were considered by Rathman and Scamehorn [10]. Treating micelle formation by small system thermodynamics, Gilányi suggested a diffuse monolayer structure instead of the idealized Goüy - Chapman model [11]. This listing can be continued up to the latest works utilizing molecular dynamics (MD) simulations [12-14].

In many cases, the electrostatic potential is considered as a key parameter governing the properties of the micellar solutions of ionic surfactants, affecting their behavior in photophysical and analytical processes, chromatog- raphy, shifting of equilibrium states and acceleration of a variety of reactions, binding various compounds, in synthesis of nanoparticles, etc. $[1-4,7,15]$. Consequently, there are many approaches to determine its value for ionic micelles.

Among the experimental methods, the most popular and universal one is based on the application of colored or fluorescent acid-base indicators [3,15-20]. There, $\Psi$ is determined using the so-called apparent dissociation constant, $K_{\mathrm{a}}^{\text {app }}$, of the indicator bound to the micelle and the so-called intrinsic constant, $K_{\mathrm{a}}^{\mathrm{i}}$, Eq. 1.,

$$
\Psi=\frac{R T \ln 10}{F} \times\left(\mathrm{p} K_{\mathrm{a}}^{\mathrm{i}}-\mathrm{p} K_{\mathrm{a}}^{\mathrm{app}}\right)
$$

Here $R, T, F$ have their usual meanings.

The apparent constant is in fact a two-phase equilibrium constant because the $\mathrm{pH}$ value is determined in the bulk phase, whereas the acid-base couple of the indicator dye is located in the micellar palisade. As the $\mathrm{p} K_{\mathrm{a}}^{\mathrm{i}}$ value, the $\mathrm{p} K_{\mathrm{a}}^{\mathrm{app}}$ value of the same indicator in micelles of nonionic surfactant is usually used. Some more sophisticated assumptions to determine these values are also used $[3,15,16,19,20]$. A similar approach is based on using acid-base equilibria of stable free radicals and the electronic spin resonance method [21]. A method of monitoring the fluorescence changes along with electrostatic potential alterations was developed for membranes and vesicles [22]. Utilization of the small-angle neutron scattering method for determination of the surface electrical charge and potential of surfactant micelles is also reported [23].

Yet, all these experimental methods are based on the use of molecular probes, which makes their results depending on the chosen 
probe. One of the reasons is the variation of the probe location in the micelle. In general, the probes are considered to be located within the Stern layer, which implies the $\Psi$ in Eq. 1 should be somewhere between $\Psi_{0}$ and $\Psi_{\delta}$. Another reason is the possibility of specific interactions between the probe and surfactant. In total, for sodium dodecyl sulfate (SDS) micelles the variation of $\Psi$, as found using Eq. 1, reaches about $200 \mathrm{mV}$ [15].

This makes the demand for approaches that consider the sole micelles and, hence, are free from this issue. In this connection, the most relevant approach is the electrophoretic measurement of the electrokinetic (or $\zeta$-) potential of colloid particles. No foreign probes are required, and the obtained $\zeta$ values characterize solely the particles and the solution. However, $\zeta$-potential is a priori of a lesser magnitude than $\Psi$ because corresponds not to the surface, but to some slipping plane located $\sim 1 \mathrm{~nm}$ far from it. Hence it is just a lower estimate of $\left|\Psi_{0}\right|$. It was supposed that the rough and dynamical character of the micelle surface due to monomer motion reduces the potential fade near the surface smearing the difference between the $\Psi$ and $\zeta$ values [24]. However, this assumption was later considered improbable because the slipping plane is located about $0.8 \mathrm{~nm}$ farther than the Stern layer [25].

So that, at present, only theoretical methods to the stated problem are available. The mostly used ones are based on solving the Poisson Boltzmann (PB) equation, Eq. 2. This equation establishes the relation between the charge density of the system and the electrostatic potential created by it. The density is represented as a sum of two contributions: the fixed one, which is constant and belongs to the solute, and the mobile one, which depends on the electrostatic potential distribution in a self-consistent manner and represents the coions and counterions in the solution [26]. It is this equation that grounds the Goüy - Chapman model of EDL, as well as the Debye - Hückel theory of strong electrolytes.

$$
\nabla \cdot(\varepsilon(x, y, z) \nabla \psi(x, y, z))=-\frac{\rho(x, y, z)}{4 \pi \varepsilon_{0}}=-\frac{\rho_{f}(x, y, z)}{4 \pi \varepsilon_{0}}+\frac{F c}{2 \pi \varepsilon_{0}} \sinh \left(-\frac{F \psi(x, y, z)}{R T}\right)
$$

Here $\psi(x, y, z)$ is the electrostatic potential, $\varepsilon_{0}$ has its usual meaning, $\rho(x, y, z)$ is total charge density, $\varepsilon(x, y, z)$ is the relative permittivity (in the general case it is a function of coordinates, too), $\rho_{f}$ is the fixed charge density, $c$ is the total concentration of the electrolyte (assumed here to consist of single-charged ions).

Overall, the solvent is treated here as a continuum characterized with uniform relative permittivity, thus, disregarding the discrete nature of solvent molecules and ions. The solute is also treated as a continuum with some other value of relative permittivity. The ions are originally considered to be point charges occupying no volume. Nevertheless, by adjusting boundary conditions the ions may be attributed with a radius $R_{i}$ for interaction with the solute making them unable to approach it tightly, as is assumed in the Stern model of EDL.

The PB equation is a non-linear differential equation of the second order, which makes its analytical solving possible just for the simplest geometries of charge distribution like uniformly charged planes, spheres, or cylinders. It can be linearized to facilitate solving, but this procedure is correct for relatively weakly 
charged solutes only. The approximate analytical solution of this equation in its non-linear form for a uniformly charged spherical particle with radius $r$ was deduced by Ohshima, Healy, and White, Eq. 3 [27]. It will be called Ohshima - Healy - White (OHW) equation further in the text.

The authors also derived the formula for cylindrical particles [27]. If the $q_{s}$ value is known, $\Psi_{0}$ can be calculated and vice versa. In summary, there are two parameters for a micelle represented as a homogeneously charged sphere: radius and molecular area of a head group. In this respect, it is worth to mention the work by Lukanov and Firoozabadi who numerically solved the PB equation modified to incorporate non-electrostatic solute - ions interactions for similar geometry [28].

In principle, Eq. 3 may be used to predict the potential of Stern layer $\Psi_{\delta}$ instead of the surface potential $\Psi_{0}$. In such a case, the surface charge density is reduced due to the presence of adsorbed counterions. Hence, the $q_{s}$ value must be multiplied by $(1-\beta)$ to reflect the par- tial neutralization of the micelle charge, where $\beta$ is the degree of neutralization. Additionally, the $r$ value should be increased to match the total size of the micelle together with its Stern layer. It was shown that for well-defined colloidal systems like aqueous micellar solutions of SDS or cetyltrimethylammonium bromide (CTAB) such calculations give plausible results $[16,17]$.

Yet, despite the usefulness of the described equation, it is a severe simplification originating from the inability to analytically solve the PB equation for a case of complex geometry. However, at present one is no more constrained to analytical solutions due to the availability of computers allowing the direct numerical solving of this equation. Nowadays there is a variety of software developed for this sake [29]. There, the input is the atomistic model of the system of interest (consisting of the positions of atoms, their van der Waals radii and atomic point charges) and, the output is the corresponding distribution of the electrostatic potential in surrounding space.

$$
\begin{array}{r}
q_{s}=\frac{2 \varepsilon_{0} \varepsilon R T \kappa}{F} \sinh (Y / 2)\left(1+\frac{2}{\kappa r \cosh ^{2}(Y / 4)}+\frac{8 \ln [\cosh (Y / 4)]}{(\kappa r)^{2} \sinh ^{2}(Y / 2)}\right)^{\frac{1}{2}} \\
q_{s}=\frac{e}{s}=e \frac{N_{a g g r}}{4 \pi r^{2}} ; \quad Y=\frac{F \Psi_{0}}{R T} ; \quad \kappa=\sqrt{\frac{2 F^{2} I}{\varepsilon_{0} \varepsilon R T}}
\end{array}
$$

Here, $q_{s}$ is the surface charge density, $s$ is the molecular area of a head group on the micellar surface, $N_{\text {aggr }}$ is the aggregation number, $e$ is the elementary charge, $\kappa$ is the reciprocal Debye length, $I$ is the ionic strength of a solution. (represented as a three-dimensional grid of $\psi(x, y, z)$ values). The solution parameters (relative permittivity, temperature, ionic strength) are also required. The main benefit of the numerical approach is that the charge distribution is considered in all its complexity with no need to simplify or impose some limitations on its geometry. Additionally, the interpolation schemes can be applied for $\varepsilon(x, y, z)$ at the solute-solvent boundary to form a smooth contact between the two continuums. Hence, 
this technique is widely used for studying various biomolecules and biological assemblies (lipid membranes, individual proteins, and protein complexes including viruses) having an elaborate structure, where it reveals the regions of high positive or negative charge density [30-33]. However, to our knowledge, such objects as surfactant micelles have not been investigated with this method up to date.

This work aims to fill this gap and perform the numerical computations of the electrostatic potential around ionic micelles based on their accurate atomistic models instead of the simplified ones. We chose SDS and CTAB micelles for this study as the typical and well-defined ones. The produced results will be contrasted with the predictions of the simplified model given by the OHW equation.

EXPERIMENT AND DISCUSSION OF THE RESULTS. Computational procedure. The parameters of micelles required by the OHW equation (radius, surface area, degree of neutralization) were calculated grounding on the molecular dynamics simulations of SDS and CTAB micelles composed from 60 (the former) or 80 and 95 (the latter) surfactant monomers. Unlike SDS, for CTAB there is some spread in the experimental data about aggregation number, hence, two values of $N_{\text {aggr }}$ were considered. We used the last 10 ns interval of the trajectories generated during our previous investigations of these systems, for details please refer to the corresponding papers $[13,14]$. The radius was calculated in two ways: 1) as the gyration radius of hydrocarbon core multiplied by $\sqrt{5 / 3}$, and 2) as the position of the maximum of the radial distribution function (RDF) between micelle center of mass and $\mathrm{S}(\mathrm{N})$ head groups atoms. Both ways provided similar values (within $0.05 \mathrm{~nm}$ ).
The solution parameters submitted to the OHW equation were: $\varepsilon=78.5 ; T=298.15 \mathrm{~K}$; $I=0.05 \mathrm{M}=50 \mathrm{~mol} \mathrm{~m}^{-3}$.

To determine the degree of neutralization we calculated the radial distribution function $g(d)$ of counterions around the micelle, Eq. 4. It shows how much the density of counterions on distance $d$ from the surface differs from the cell-average value. Preliminary, the function $N(d)$ was calculated as the total number of ions laying within distance $d$ from the surface. The peaks on RDF's indicate the regions of increased ions density, hence, it is reasonable to define the Stern layer as the peak in $g(d)$.

$$
\begin{aligned}
g(d) & =\frac{\rho(d)}{\rho_{0}}=\frac{1}{\rho_{0}} \frac{d N(d)}{d V(d)}= \\
& =\frac{1}{\rho_{0}} \frac{1}{4 \pi(r+d)^{2}} \frac{d N(d)}{d d}
\end{aligned}
$$

Where $\rho_{0}$ is the cell-average number density of counterions, $\rho(d)$ is the number density of counterions at distance $d$ from micelle, $d N$ is the number of counterions within an infinitely thin layer around micelle at distance $d, d V$ is the volume of this layer, which is approximated by the volume of a spherical layer, $r$ is the micelle radius.

The input for $\mathrm{PB}$ computations was obtained in two stages. At first, the distribution of electrostatic potential around micelles was calculated using the Adaptive Poisson - Boltzmann solver (APBS) software $[34,35]$. The box size was chosen similar to the MD cell size, namely $8 \mathrm{~nm}$ for SDS and $10 \mathrm{~nm}$ for CTAB, and grid dimensions were $257 \times 257 \times 257$. The micelle was centered in the box. Relative permittivity was set to 78.5 for water and 2 for micelle (matching higher alkanes like $n$-decane). Radius of solvent molecules was set to the 
default value that is $0.14 \mathrm{~nm}$. Because we deal with highly charged systems, non-linearized version of Poisson-Boltzmann equation was chosen. Both the cation and the anion of the background electrolyte were single-charged and were attributed with radius $0.1 \mathrm{~nm}$ for SDS and $0.2 \mathrm{~nm}$ for $\mathrm{CTAB}$ matching the ionic radii of $\mathrm{Na}^{+}$and $\mathrm{Br}^{-}$, respectively [36]. The computations were carried out at a range of electrolyte concentrations from $0.05 \mathrm{M}$ to $1 \mathrm{M}$ including the case of no electrolyte.

The atomistic models were obtained from the above stated MD simulations of SDS and CTAB micelles. For each surfactant, 3 models were prepared by extracting instantaneous configurations from MD trajectory at $10 \mathrm{~ns}$, $15 \mathrm{~ns}$, and $20 \mathrm{~ns}$. The atomic point charges were taken from the used potential models, which belong to the optimized potentials for liquid simulations - all-atom (OPLS-AA) force field $[13,14]$. The atomic radii were provided by $g m x$ editconf utility executed with -mead option specified.

The second stage was averaging the spatial distribution of the electrostatic potential $\psi(x, y, z)$ over relevant regions to get meaningful average values, which can be interpreted and compared with predictions of other methods. The quantities predicted by analytical models of micelles are usually related to the distance from the micelle surface, while the micelle shape itself is considered as a simple geometric form (sphere, cylinder etc.). However, in our case it appears non-trivial to define the concept of "distance from micelle surface" because the atomistic models have rough, irregular shapes preventing the application of simple geometric criteria like distance from the micelle center of mass. Consequently, it is difficult to outline the regions for averaging the $\psi(x, y, z)$.

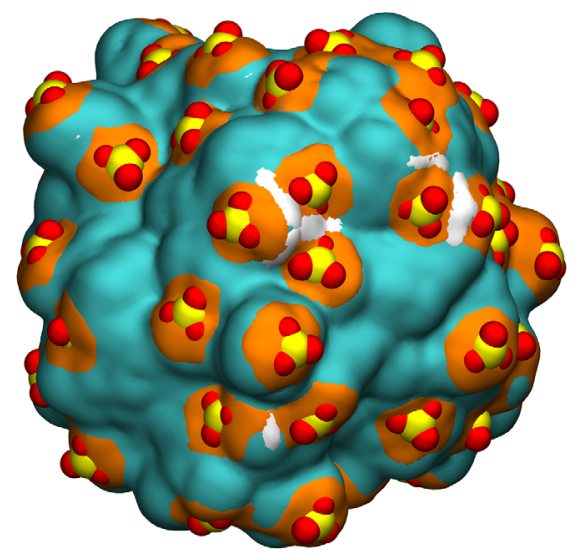

B

Fig. 1. A: The principle of calculation of solvent-accessible surfaces. Dark blue circles $-\mathrm{CH}_{2}$ groups of hydrocarbon core, dotted orange circle - a head group, red line "vdW" - van der Waals surface of hydrocarbon core, light blue outlined circles - solvent probes with radius $R_{w}$, light green outlined circle solvent probe with radius $\left(R_{W}+x\right)$, solid and dashed violet lines - SAS of hydrocarbon core obtained with the smaller and the bigger probes, respectively. The former SAS establishes the origin of the distance scale, $d=0$, and the points of the latter SAS are considered as located on distance $x$ from the hydrocarbon core.

B: SDS micelle within its solvent-accessible surface. The regions of the surface located within $0.4 \mathrm{~nm}$ of $\mathrm{S}$ atoms are highlighted orange, and the regions within $0.5 \mathrm{~nm}$ of two $\mathrm{S}$ atoms are highlighted white. $\mathrm{S}$ and $\mathrm{O}$ atoms are shown as red and yellow spheres, respectively. 
We solved this problem by employing solvent-accessible surfaces (SAS). According to the definition, SAS is the locus of the center of a spherical probe as it rolls over the accessible regions of the van der Waals surface of object. The probe radius $R$ is usually set to the approximate radius of water molecules $R_{W}$ that is 0.14 $\mathrm{nm}$. In turn, the van der Waals (also called molecular) surface is the union of atom-centered spheres each having the radius set to the van der Waals radius of the corresponding atom [37]. Here we generated the solvent-accessible surface of the hydrocarbon core of micelles using the gmx sasa utility with the default value of the probe radius $(0.14 \mathrm{~nm})$ and increased precision (parameter -dots 100). The head groups $\mathrm{OSO}_{3}^{-}$and $\mathrm{CH}_{2} \mathrm{~N}\left(\mathrm{CH}_{3}\right)_{3}^{+}$were omitted in the calculation as not forming the surface but protruding from it. This SAS was considered as the actual micelle surface, and it set the origin for measuring distance $d$ towards a bulk solution: all points of this SAS were attributed with $d=0$. Further, a series of SAS was generated using larger probe radii of $(0.14+x) \mathrm{nm}$ where $x$ ranged from 0.05 to $1 \mathrm{~nm}$. These surfaces were larger than the original one and represented an accurate equivalent to the notion "the place of points at distance $x$ from micelle". The principle is illustrated in Figure 1A.

These surfaces were used then as the regions for averaging the electrostatic potential distribution. The average of $\psi(x, y, z)$ evaluated over the surface generated using probe radius $(0.14+x)$ $\mathrm{nm}$ was considered as the $\Psi$ value at distance $d=x$ from micelle surface. Because each surface was intrinsically represented as a set of points, and the potential distribution was a three-dimensional grid, calculation of the average value was done by enumerating the surface points and summing up the values of potential there, which were in turn determined via trilinear interpolation between the neighboring grid points. Importantly, not all surface points were used here, but only a subset: we excluded the SAS points located closer than $0.4 \mathrm{~nm}$ to any $\mathrm{S}(\mathrm{N})$ atom of head groups and the points within $0.5 \mathrm{~nm}$ of two or more $\mathrm{S}(\mathrm{N})$ atoms at the same time. The justification is provided in the Results section below. An example of a SAS with the indication of the excluded regions is shown in Figure 1B.

The parameters of micelles and subsequent solutions of OHW equation

\begin{tabular}{c|c|c|c|c|c|c}
\hline \hline surfactant & $N_{\text {aggr }}$ & $r, \mathrm{~nm}$ & $s, \mathrm{~nm}^{2}$ & $\left|\Psi_{0}\right|, \mathrm{mV}$ & $\beta$ & $\left|\Psi_{\delta}\right|, \mathrm{mV}$ \\
\hline SDS & 60 & 1.81 & 0.686 & 139 & 0.48 & 94 \\
CTAB & 80 & 2.22 & 0.774 & 134 & 0.61 & 75 \\
CTAB & 95 & 2.35 & 0.731 & 138 & 0.65 & 74 \\
\hline \hline
\end{tabular}

Table 2

Electrostatic potential of micelles from numerical solution of PB equation at $I=0.05 \mathrm{M}$

\begin{tabular}{c|c|c|c|c|c}
\hline \hline surfactant & $N_{a g g r}$ & $\left|\Psi_{0}\right|, \mathrm{mV}$ & $|\Psi(0.2)|, \mathrm{mV}$ & $|\Psi(0.4)|, \mathrm{mV}$ & $\left|\Psi_{\delta}\right|, \mathrm{mV}$ \\
\hline SDS & 60 & 100 & 85 & 68 & 75 \\
CTAB & 80 & 149 & 106 & 76 & 70 \\
CTAB & 95 & 151 & 111 & 85 & 78 \\
\hline \hline
\end{tabular}


Firstly, we focus on the theoretical predictions of the OHW equation. The parameters of micelles obtained from MD simulations $(r$, $s, \beta)$ and the subsequent solutions of Eq. 2 are collected in Table 1. For $\Psi_{\delta}$ calculation, the micelle radius $r$ was increased by $0.1 \mathrm{~nm}$ in order to reflect the incorporation of neighboring ions (the $s$ value was accordingly changed, too). We deliberately chose the addition less than $\delta$ because the visual examination of MD trajectories revealed that most of these counterions are located in between the head groups or alongside them, therefore, do not advance far towards a bulk solution. The analysis of $g(d)$ graphs suggested placing the Stern layer boundary $\delta$ at about $0.32 \mathrm{~nm}$ for SDS and $0.45 \mathrm{~nm}$ for CTAB, Figure 2. The corresponding $\beta$ values found as $N(\delta) / N_{a g g r}$ are 0.48 and 0.61 , respectively. We note that the experimentally determined $\beta$ values vary within the range of $0.5-0.9$ for different surfactants and depend on the method used $[3,15]$.

There is no significant difference found between $\Psi$ values of CTAB micelles composed of

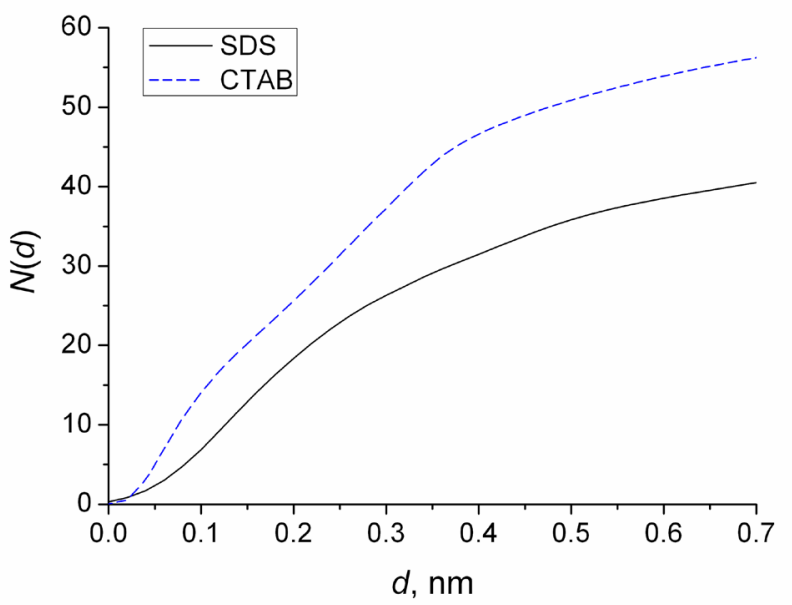

A
80 and 95 monomers indicating the negligible effect of the micelle size in this case.

Now we turn to the results of PB calculations. From the qualitative point of view, visual examination of the electrostatic potential distribution depicted as equipotential surfaces (isosurfaces) reveals its complex shape. $\psi(x, y, z)$ is quite uniform near the regions where the hydrocarbon core is exposed to water, however, it sharply increases in magnitude upon approaching the head groups. Its gradient increases there, too, as is evidenced by a tighter spacing between isosurfaces: there the magnitude reaches $\geq 260 \mathrm{mV} / \mathrm{nm}$, while far from head groups it drops to $\leq 80 \mathrm{mV} / \mathrm{nm}$.

To get quantitative results we averaged $\psi(x, y, z)$ following the procedure described in the Computational procedure section. The previous observations motivated us to exclude the vicinities of head groups from averaging: these regions, while having comparatively small total area, are located at $\psi(x, y, z)$ values very different from the rest of the surface. Therefore, keeping them would distort the average.

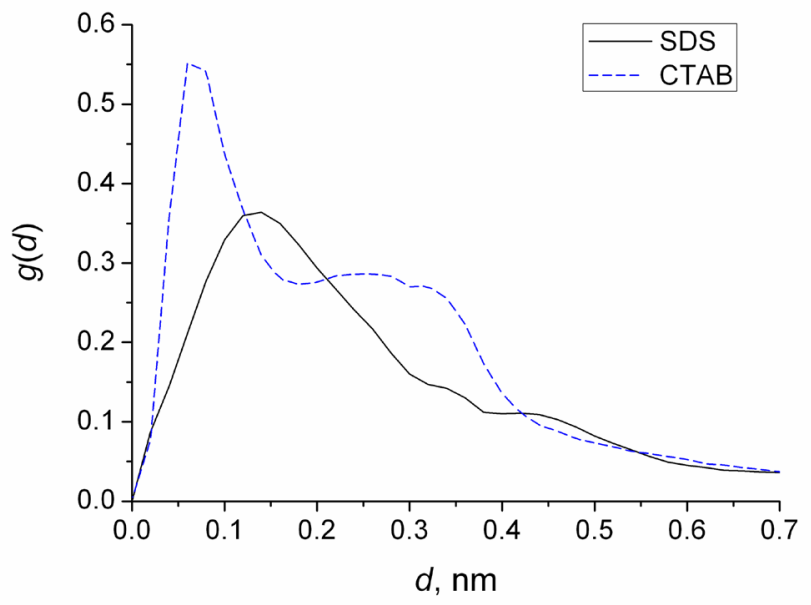

$\mathrm{B}$

Fig. 2. A: the number of counterions at given distance from micelle surface; B: radial distribution function of counterions around the micelle. The origin corresponds to the solvent-accessible surface of hydrocarbon core. 
The produced dependences of $\Psi$ on distance from micelle surface and ionic strength are presented in Figures 3, 4A and summarized in Table 2. The profiles demonstrate the expected behavior of gradual monotonic decrease towards zero with distance and ionic strength. At similar conditions, $\Psi$ around CTAB micelles have a larger magnitude than around the SDS ones. It should be emphasized that the data for high ionic strengths is rather evaluative because all the computations were

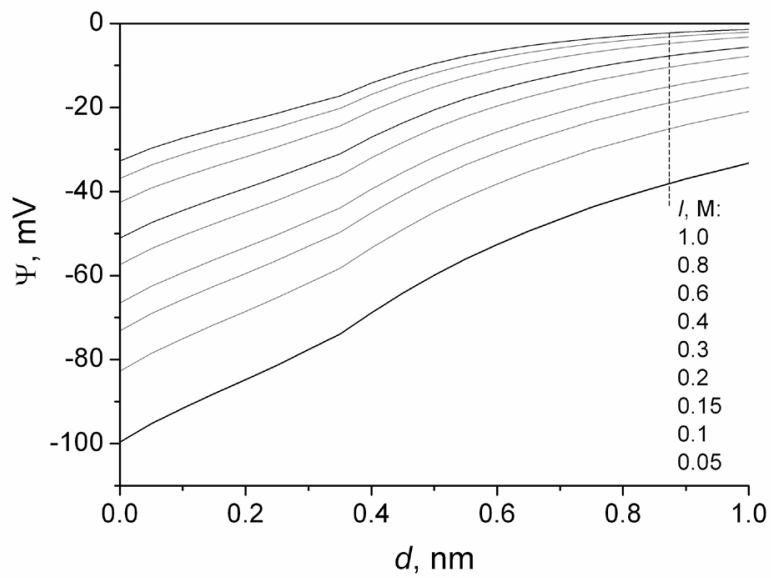

$\mathrm{A}$ done using the same atomistic model. While at such conditions the micelles usually change shape from ellipsoidal to rod-like.

A feature of the numerical computations is the possibility to handle the case of a "bare" micelle in the absence of counterions and background electrolyte. In this case $I=0$ and $\kappa^{-1}$ tends to infinity preventing the use of the OHW equation. The estimated $\Psi_{0}$ values equaled $-535 \mathrm{mV}$ and $+630 \mathrm{mV}$ for SDS and CTAB micelles, respectively.

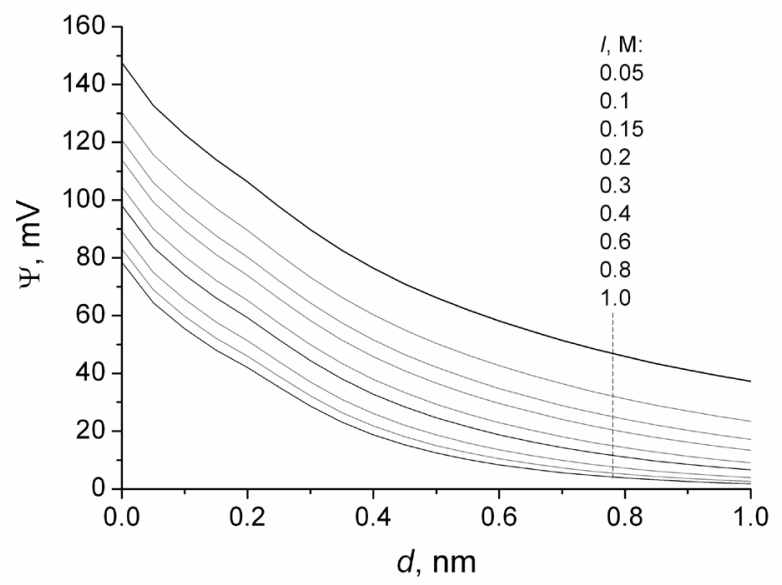

$\mathrm{B}$

Fig. 3. Profiles of electrostatic potential fade with distance for SDS (A) and CTAB (B) micelles at different ionic strengths.

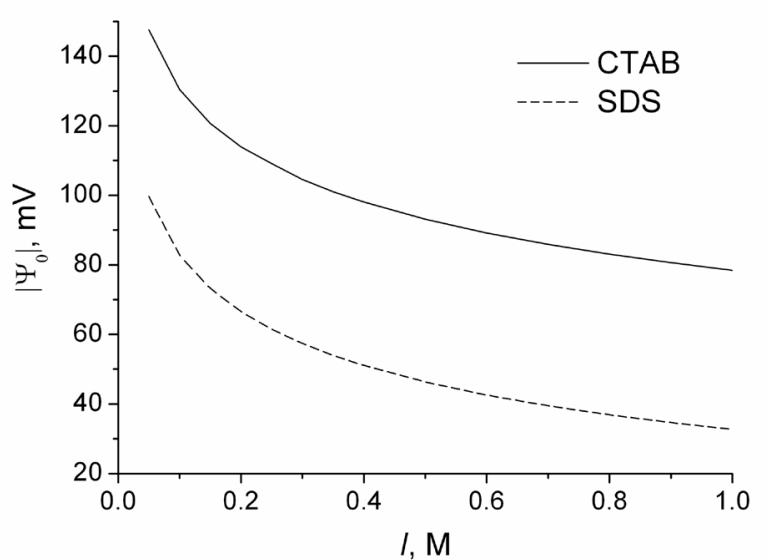

A

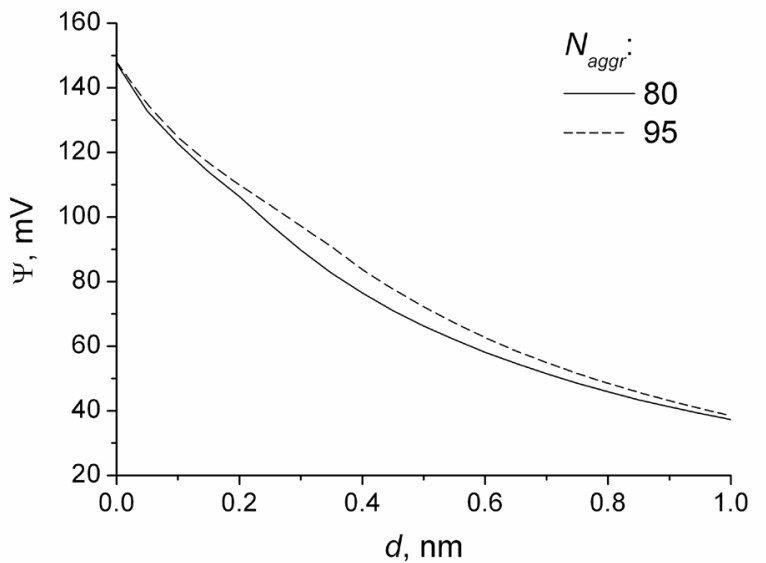

$\mathrm{B}$

Fig. 4. A: Dependence of surface electrostatic potential on ionic strength for SDS and CTAB micelles; B: Profile of electrostatic potential fade with distance for CTAB micelles of different sizes at $I=0.05 \mathrm{M}$. 
Table 3

\section{Effect of counterions radius on $\Psi_{0}$ from the numerical solution of PB equation} at $I=0.05 \mathrm{M}$. The original values are highlighted in bold

\begin{tabular}{l|l|l|l|l}
\hline \hline \multirow{2}{*}{ surfactant } & \multirow{2}{*}{$N_{a g g r}$} & \multicolumn{4}{|c}{$\left|\Psi_{0}\right|, \mathrm{mV}$} \\
\cline { 3 - 5 } & & $R_{i}=0.2 \mathrm{~nm}$ & $R_{i}=0.1 \mathrm{~nm}$ & $R_{i}=0$ \\
\hline SDS & 60 & 130 & $\mathbf{1 0 0}$ & 71 \\
CTAB & 80 & $\mathbf{1 4 9}$ & 106 & 74 \\
\hline \hline
\end{tabular}

To check the robustness of the presented results, we varied several factors, which can affect the computed $\psi(x, y, z)$ distributions.

The graphs presented above were produced, basing on a single model for each micelle. To estimate the effect of the choice of configuration, we repeated the computations at $I=0.05 \mathrm{M}$ using two other models. The produced $\Psi$ profiles were within $5 \mathrm{mV}$ at $d=0$ and within $3 \mathrm{mV}$ for larger $d$ indicating that using a single model is enough to get a correct result. The possible reason is that the main structural motifs of the micelle surface are observed at any time during the evolution of its shape. We note that the values in Table 2 are averages over three models.

Next, because of the uncertainty with $N_{\text {aggr }}$ of CTAB micelles we repeated the computations using the models with $N_{\text {aggr }}=95$ instead of 80. Both results are confronted in Table 2 and Figure $4 \mathrm{~B}$. It is seen that while some difference is present, the profiles agree well: the $\Psi$ values almost coincide at the shortest $(\leq 0.15 \mathrm{~nm})$ and longest $(\geq 0.7 \mathrm{~nm}) d$ values. In between the discrepancy is no more than $10 \mathrm{mV}$ that is moderate. This is in line with the behavior of the OHW equation.

A crucial factor is the radius of ions of the background electrolyte. We tested the values $0.2 \mathrm{~nm}$ for SDS (instead of $0.1 \mathrm{~nm}$ ) and $0.1 \mathrm{~nm}$ for CTAB (instead of $0.2 \mathrm{~nm}$ ). Also, the point- like case $\left(R_{i}=0\right)$ was checked. Unlike the previous factors, here the influence appeared significant for both kinds of micelles: each decrease of $R_{i}$ by $0.1 \mathrm{~nm}$ reduced $\left|\Psi_{0}\right|$ by $30-40 \mathrm{mV}$, Table 3 . This effect is expected due to the appearance of counterions closer to the surface.

Finally, we tested the influence of the computation parameters. The box size increase to $12 \times 12 \times 12 \mathrm{~nm}^{3}$ and the grid dimensions decrease to $129 \times 129 \times 129$ were found to cause the negligible difference of $\Psi_{0}(<3 \mathrm{mV})$.

First of all, the comparison of results in Tables 1 and 2 shows that both OHW equation and numerical computations provide the magnitude of $\Psi_{0}$ in the range of $100-150 \mathrm{mV}$ for both kinds of micelles. The closer look, however, reveals a discrepancy: while OHW equation predicts almost equal $\left|\Psi_{0}\right|$ for SDS and CTAB (within $5 \mathrm{mV}$ ), their numerical results are much different (by $\sim 50 \mathrm{mV}$ ). This behavior seems rather surprising because both ways are based on the same theory (that is Eq. 2) and requires explanation.

The main distinction between the ways is the geometry of the model: while the OHW equation represents the micelle with head groups as a smooth sphere with the uniformly distributed charge, in numerical computations the protrusion of head groups from the hydrocarbon core and their discrete charge is taken into account. 
The second distinction is more subtle but no less important: in the derivation of the $\mathrm{OHW}$ equation, the ions were treated as point charges implying they approach infinitely close to the solute. Oppositely, the used software for numerical solution allowed tuning the distance of the closest approach between the ions and the solute in terms of ions radii $R_{i}$. As was mentioned before, this parameter strongly affects the results. The examination of Table 3 shows that if equal radii are used, the $\Psi_{0}$ values of SDS and CTAB become much closer (within $7-20 \mathrm{mV}$ ) resembling the behavior of the OHW equation.

Still, there remains the difference in predicted $\Psi_{0}$ magnitude. Surprisingly, the closest match between $\mathrm{OHW}$ and numerical predictions is observed for $R_{i}=0.2 \mathrm{~nm}$ instead of zero size. We attribute this fact to the geometry of the models via the following qualitative consideration. The discrete head groups have a high charge density. When their charge is smeared across the whole surface, the charge density proportionally decreases. As a result, such uniformly charged surface exerts proportionally weaker attraction to counterions. This, however, leads to exponentially smaller concentration and charge density of the approached counterions. Therefore the original surface charge becomes neutralized to a lesser extent resulting in higher $\left|\Psi_{0}\right|$ values. To achieve the same magnitude in numerical computation one has to artificially prevent counterions from accumulating around highly charged head groups by attributing the ions with $\sim 0.2 \mathrm{~nm}$ radii.

Considering the surface potential of "bare" micelles without counterions, its large magnitude against the $\left|\Psi_{0}\right|$ values in Table 2 indicates the effect exerted by the outer part of EDL.
Turning to the potential of Stern layer $\Psi_{\delta}$, both ways agree that for CTAB it equals $\sim 75 \mathrm{mV}$. For SDS the numerical result is $-75 \mathrm{mV}$. Its direct comparison with the OHW solution is impossible because of the discrepancies described above.

To summarizing, in our opinion the distinction of results of the used ways is caused by the different treatment of head groups and counterions. Among the two ways, we have a preference for the numerical solution because it implies fewer simplifications and assumptions and, thus, provides a more realistic treatment of the examined systems. Nevertheless, the analytical equation produces the values of correct magnitude with much less computational effort.

CONCLUSIONS. The surface electrostatic potential $\Psi_{0}$ of micelles of common surfactants sodium dodecyl sulfate and cetyltrimethylammonium bromide was estimated by two theoretical approaches based on the Poisson - Boltzmann equation. The analytical equation derived for uniformly charged spherical particles and point-like counterions was found to provide similar $\Psi_{0}$ values for both micelles. Oppositely, the numerical solution with the geometry of the systems accurately reproduced and ions attributed with radii equal to their ionic radii resulted in pronouncedly higher $\Psi_{0}$ of CTAB micelles than of SDS ones. Namely, at background electrolyte ionic strength of $0.05 \mathrm{M}$ the values equaled $+150 \mathrm{mV}$ and $-100 \mathrm{mV}$, respectively. By the same approach, the electrostatic potential on the boundary of the Stern layer $\Psi_{\delta}$ was found to be ca. $+74 \mathrm{mV}$ and $-75 \mathrm{mV}$, respectively. The discrepancy between the approaches was explained on the basis of the assumptions and simplifications made in them. 
ACKNOWLEDGEMENTS. The authors thank the Ministry of Education and Science of Ukraine for financial support in the framework of the project "Novel nanomaterials based on the lyophilic self-assembled systems: theoretical prediction, experimental investigation, and biomedical applications" (0120U101064).

КОНТИНУАЛЬНО-ЕЛЕКТРОСТАТИЧНЕ ДОСЛДЖЕННЯ ЙОННИХ МШЦЕЛ ІЗ ВИКОРИСТАННЯМ АТОМІСТИЧНИХ МОДЕЛЕЙ

\section{В. С. Фарафонов ${ }^{*}$ О. В. Лебідь, М. О. Мчедлов-Петросян}

Харківський національний університет імені В. Н. Каразіна, пл. Свободи, 4, Харків, 61022, Україна

*e-mail:farafonov@karazin.ua

Розглянуто питання про ключовий параметр, пов'язаний з будовою подвійного електричного шару міцел йоногенних ПАР, - електростатичний потенціал. Наведено короткий огляд експериментальних методів та теоретичних моделей для оцінки електростатичного потенціалу. Запропоновано метод розрахунку електростатичного потенціалу, який базується на чисельному розв'язанні рівняння Пуассона - Больцмана, з використанням атомістичної моделі міцели йоногенної ПАР. Необхідні для побудови атомістичних моделей параметри одержано з молекулярно-динамічного моделювання. Із використанням цього методу розраховано електростатичний потен- ціал для міцел додецилсульфату натрію та броміду цетилтриметиламонію при різних іонних силах. Результати обговорюють порівняно зі значеннями, розрахованими в рамках спрощеної моделі за рівнянням Ошими - Хілі - Уайта.

Ключові слова: міцела поверхнево-активної речовини, шар Штерна, електростатичний потенціал, рівняння Пуассона - Больцмана, доступна для розчинника поверхня.

\section{REFERENCES}

1. Surfactants science and technology. Retrospects and Prospects. Ed. Romsted L. S. Boca Raton: CRC Press. 2014.

2. Handbook of Surface and Colloid Chemistry. Ed. Birdi K. S. Boca Raton: CRC Press, 2009.

3. Mchedlov-Petrossyan N. O., Vodolazkaya N. A., Kamneva N. N. Acid-base equilibrium in aqueous micellar solutions of surfactants. In: Micelles: Structural Biochemistry, Formation and Functions \& Usage. Ed. Bradburn D., Bittinger J. N. Y.: Nova Publishers. 2013. Chapter 1.1-71.

4. Rusanov A. I. Micellization in Surfactant Solutions. Reading: Harwood Academic Publishes. 1997.

5. Rusanov A. I. Nanothermodynamics: chemical approach. Russian Chemistry Journal. 2006. 50 (2): 145-151 [in Russian].

6. Shinoda K. The significance and characteristics of organized solutions. Journal of Physical Chemistry. 1985. 89 (11): 24292431. https://doi.org/10.1021/j100257a055. 
7. Organized Solutions. Surfactants in Science and Technology. Ed. Friberg S. E., Lindman B. N.Y.: Marcel Dekker. 1992.

8. Stigter D. On the Adsorption of Counterions at the Surface of Detergent Micelles. Journal of Physical Chemistry. 1964. 68 (13): 3603-3611. https://doi.org/10.1021/ j100794a028.

9. Stigter D. Micelle formation by ionic surfactants. III Model of Stern layer, ion distribution, and potential fluctuation. Journal of Physical Chemistry. 1975. 79 (10): 1008-1013. https://doi.org/10.1021/ j100577a013.

10. Rathman J. F., Scamehorn J. F. Counterion binding on mixed micelles. Journal of Physical Chemistry. 1984. 88 (24): 5807-5816. https://doi.org/10.1021/j150668a014.

11. Gilányi T. Fluctuating micelles: a theory of surfactant aggregation 2. Ionic surfactants. Colloids and Surfaces A: Physicochemical and Engineering Aspects. 1995. 104 (1): 119-126. https://doi.org/10.1016/09277757(95)03223-Z.

12. Roussel G., Michaux C., Perpete E. A. Multiscale molecular dynamics simulations of sodium dodecyl sulfate micelles: from coarse-grained to all-atom resolution. Journal of Molecular Modeling. 2014. 20: 2469-2476. https://doi.org/10.1007/ s00894-014-2469-0.

13. Farafonov V. S., Lebed A. V. Developing and validating a set of all-atom potential models for sodium dodecyl sulfate. Journal of Chemical Theory and Computation. 2017. 13 (6): 2742-2750. https://doi. org/10.1021/acs.jctc.7b00181.

14. Farafonov V. S., Lebed A. V. Molecular dynamics simulation study of cetylpyridinum chloride and cetyltrimethylammoni- um bromide micelles. Kharkov University Bulletin, Chemistry Series. 2016. 27 (50): 25-30. https://doi.org/10.26565/2220637X-2017-27-03.

15. Mchedlov-Petrossyan N. O. Protolytic equilibrium in lyophilic nano-sized dispersions: Differentiating influence of the pseudophase and salt effects. Pure and $A p$ plied Chemistry. 2008. 80 (7): 1459-1510. https://doi.org/10.1351/pac200880071459.

16. Hartland G.V., Grieser F., White L.R. Surface potential measurements in pentanol-sodium dodecyl sulphate micelles. Journal of Chemical Society, Faraday Transactions. 1978. 1 (83): 591-613. https://doi. org/10.1039/F19878300591.

17. Mchedlov-Petrossyan N. O., Vodolazkaya N. A., Yakubovskaya A. G., Grigorovich A.V., Alekseeva V. I., Savvina L. P. A novel probe for determination of electrical surface potential of surfactant micelles: N,N'-di-n-octadecylrhodamine. Journal of Physical Organic Chemistry. 2007. 20 (5): 332-344. https://doi.org/10.1002/ poc. 1150 .

18. Fernandez M. S., Fromherz P. Lipoid pH indicators as probes of electrical potential and polarity in micelles. Journal of Physical Chemistry. 1977. 81 (18): 1755-1761. https://doi.org/10.1021/j100533a009.

19. Funasaki N. Micellar effects on the kinetics and equilibrium of chemical reactions in salt solutions Journal of Physical Chemistry. 1979. 83 (15): 1998-2003. https://doi. org/10.1021/j100478a014.

20. Grieser F., Drummond C. J. The physicochemical properties of self-assembled surfactant aggregates as determined by somemolecular spectroscopic probe techniques. Journal of Physical Chemistry. 
1988. 92 (20): 5580-5593. https://doi. org/10.1021/j100331a012.

21. Khlestkin V. K., Polienko J. F., Voinov M. A., Smirnov A. I., Chechik V. Interfacial Surface Properties of Thiol-Protected Gold Nanoparticles: A Molecular Probe EPR Approach. Langmuir. 2008. 24 (3): 609612. https://doi.org/10.1021/la702823n.

22. Voinov M. A., Kirilyuk I. A., Smirnov A. I. Spin-labeled $\mathrm{pH}$-sensitive phospholipids for interfacial pka determination: synthesis and characterization in aqueous and micellar solutions. Journal of Physical Chemistry B. 2009. 113 (11): 3453-3460. https://doi.org/10.1021/jp810993s.

23. Bulavin L. A., Garamus V. M., Karmazina T. V., Pivnenko E. N. Measurements of structural and electrostatic parameters and surface tension of micelles of an ionic surfactant versus concentration, ionic strength of solution and temperature by small-angle neutron scattering. Colloids and Surfaces A: Physicochemical and Engineering Aspects. 1998. 131 (1-3): 137-144. http://dx.doi. org/10.1016/S0927-7757 (96)03882-4.

24. Aniansson G. E. A. Dynamics and structure of micelles and other amphiphile structures. Journal of Physical Chemistry. 1978. 82 (26): 2805-2808. https://doi. org/10.1021/j100515a011.

25. Lyklema J. Surface charges and electrokinetic charges: distinctions and juxtapositionings. Colloids and Surfaces A: Physicochemical and Engineering Aspects. 2011. 376 (1-3): 2-8. https://doi.org/10.1016/j. colsurfa.2010.09.021.

26. Cai Q., Hsieh M. J., Wang J., Luo R. Performance of nonlinear finite-difference Poisson - Boltzmann solvers. Journal of Chemical Theory and Computation. 2010. 6 (1):
203-211. https://dx.doi.org/10.1021\%2Fct900381r.

27. Ohshima H., Healy T. W., White L. R. Accurate analytic expressions for the surface charge density/surface potential relationship and double-layer potential distribution for a spherical colloidal particle. Journal of Colloid and Interface Science. 1980.90 (1): 17-26. https://doi.org/10.1016/00219797(82)90393-9.

28. Lukanov B., Firoozabadi A. Specific ion effects on the self-assembly of ionic surfactants: a molecular thermodynamic theory of micellization with dispersion forces. Langmuir. 2014. 30 (22): 6373-6383. https://doi.org/10.1021/la501008x.

29. Li C., Li L., Petukh M., Alexov E.. Progress in developing Poisson-Boltzmann equation solvers. Molecular Based Mathematical Biology. 2013. 1 (1): 42-62. https://dx. doi.org/10.2478\%2Fmlbmb-2013-0002.

30. Schweke H., Mucchielli M.-H., Sacquin-Mora S., Bei W., Lopes A. Protein interaction energy landscapes are shaped by functional and also non-functional partners. Journal of Molecular Biology. 2020. 432 (4): 1183-1198. https://doi. org/10.1016/j.jmb.2019.12.047.

31. Tarasova E., Farafonov V., Taiji M., Nerukh D. Details of charge distribution in stable viral capsid. Journal of Molecular Liquids. 2018. 265: 585-591. https://doi. org/10.1016/j.molliq.2018.06.019.

32. Tarabara U., Vus K., Girnyk S., Kamneva N., Lavryk O., Mikhailyuta M., Trusova V., Gorbenko G. Effect of amyloid fibrils on electrokinetic properties of lipid vesicles. East European Journal of Physics. 2017. 4 (2): 19-28. https://doi.org/10.26565/23124334-2017-2-0. 
33. Callenberg K. M., Choudhary O. P., de Forest G. L., Gohara D. W., Baker N. A., Grabe M. APBSmem: a graphical interface for electrostatic calculations at the membrane. PLOS One. 2010.5 (9): e12722. https://doi. org/10.1371/journal.pone.0012722

34. Baker N. A., Sept D., Joseph S., Holst M. J., McCammon J. A. Electrostatics of nanosystems: application to microtubules and the ribosome. Proceedings of the $\mathrm{Na}$ tional Academy of Sciences of the U.S.A. 2001. 98 (18): 10037-10041. https://doi. org/10.1073/pnas.181342398.

35. Jurrus E., Engel D., Star K., Monson K., Brandi J., Felberg L. E., Brookes D. H., Wil- son L., Chen J. Improvements to the APBS biomolecular solvation software suite: Improvements to the APBS Software Suite. Protein Science. 2017. 27 (1): 112-128. https://doi.org/10.1002/pro.3280.

36. Shannon R. D. Revised effective ionic radii and systematic studies of interatomie distances in halides and chaleogenides. Acta Crystallographica. 1976. A32: 751-767. https://doi.org/10.1107/ S0567739476001551.

37. Connolly M. Analytical molecular surface calculation. Journal of Applied Crystallography. 1983. 16 (5): 548-558. https://doi. org/10.1107/S0021889883010985.

Стаття надійшла 12.07.2021. 